\title{
Leading Neutron Production in DIS at HERA-II
}

\author{
Vitaliy Dodonov \\ Max-Planck-Institut für Kernphysik \\ Saupfercheckweg 1, D-69117 Heidelberg, Germany
}

\begin{abstract}
The semi-inclusive reaction $e p \rightarrow e^{\prime} n X$ with a final state neutron carrying a large fraction of the incoming proton energy, $x_{L}>0.32$, and transverse momentum of the neutron $p_{T}<0.2 \mathrm{GeV}$, was studied with the H1 detector at HERA in deep inelastic scattering for exchanged photon virtualities $6 \mathrm{GeV}^{2}<Q^{2}<100 \mathrm{GeV}^{2}$ and Bjorken scaling variable $1.5 \cdot 10^{-4}<x<3 \cdot 10^{-2}$. The leading neutron structure function, $F_{2}^{L N(3)}\left(Q^{2}, x, x_{L}\right)$, is measured using data taken in the years 2006 and 2007. In the pion exchange picture, leading neutron data are used to estimate the pion structure function.
\end{abstract}

\section{Introduction}

Events with a neutron carrying a large fraction of the proton beam energy have been observed in $e^{+} p$ scattering at HERA [2,3]. The mechanisms for leading neutron production are not completely understood. They may be the result of hadronisation of the proton remnant,
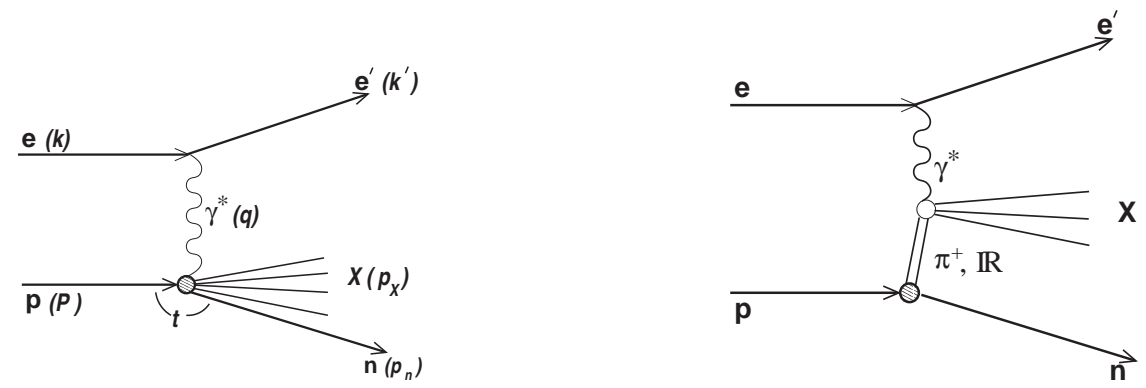

Figure 1: A generic diagram for the leading neutron production process $e p \rightarrow e^{\prime} n X$ (left) and (right) a diagram of the same process assuming that it proceeds via pion or reggeon exchange.

leaving a neutron in the final state, as shown in the diagram in Fig. 1, left. Exchange of virtual particles is also expected to contribute, as shown in the diagram in Fig. 1, right. In this model, the beam proton fluctuates into a virtual meson-neutron state. The meson (most notably $\pi^{+}$) interacts with the projectile lepton, leaving the leading neutron in the final state. The pion exchange mechanism is expected to dominate the leading neutron production at values of $x_{L} \sim 0.7-0.9$ and $p_{T} \lesssim 0.3 \mathrm{GeV}, p_{T}$ being the transverse momentum of the neutron.

Here a new measurement by the H1 Collaboration of leading neutron production in deep inelastic scattering (DIS) [4] is presented. 


\section{Data analysis}

The measurements are performed using $e^{+} p$ data collected in the years 2006 and 2007 with the $\mathrm{H} 1$ detector at a positron beam energy $E_{e}=27.6 \mathrm{GeV}$ and a proton beam energy $E_{p}=$ $920 \mathrm{GeV}$. The corresponding integrated luminosity is $122 \mathrm{pb}^{-1}$. The DIS measurements in the $Q^{2}$ range from $6 \mathrm{GeV}^{2}$ to $100 \mathrm{GeV}^{2}$ are performed with the positrons scattered into the acceptance of the Spaghetti Calorimeter, which corresponds to the polar angle range of the scattered positron $153^{\circ}<\theta<178^{\circ}$. Leading neutrons are detected in the Forward Neutron Calorimeter, which is placed $106 \mathrm{~m}$ from the interaction point at a polar angle of $0^{\circ}$ in the proton beam direction. The transverse momentum of the neutron $p_{T}$ is restricted to below $0.2 \mathrm{GeV}$, in order to reduce the contribution from non-pion exchange processes.

The kinematic variables $x, Q^{2}$ and $y$ are used to describe the inclusive DIS process. They are defined as:

$$
x=\frac{-q^{2}}{2 p \cdot q} ; \quad Q^{2}=-q^{2} ; \quad y=\frac{p \cdot q}{p \cdot k},
$$

where $p, k$ and $q$ are the four-momenta of the incident proton, the incident positron and the exchanged virtual photon, respectively.

The kinematic variables used to describe the final state neutron are:

$$
t=\left(p-p_{n}\right)^{2} \simeq-\frac{p_{T}^{2}}{x_{L}}-\left(1-x_{L}\right)\left(\frac{m_{n}^{2}}{x_{L}}-m_{p}^{2}\right) ; \quad x_{L}=1-\frac{q \cdot\left(p-p_{n}\right)}{q \cdot p} \simeq E_{n} / E_{p}
$$

where $m_{p}$ is the proton mass, $p_{n}$ is the four-momentum of the final state neutron, $m_{n}$ is the neutron mass and $E_{n}$ and $p_{T}$ are the neutron energy and transverse momentum, respectively. Thus defined, $t$ corresponds to the squared four-momentum transferred between the incident proton and the final state neutron.

The semi-inclusive structure function $F_{2}^{L N(3)}$, measured in this analysis, is defined by:

$$
\frac{\mathrm{d}^{3} \sigma(e p \rightarrow e n X)}{\mathrm{d} Q^{2} \mathrm{~d} x \mathrm{~d} x_{L}}=\frac{4 \pi \alpha^{2}}{x Q^{4}}\left(1-y+\frac{y^{2}}{2}\right) F_{2}^{L N(3)}\left(Q^{2}, x, x_{L}\right)
$$
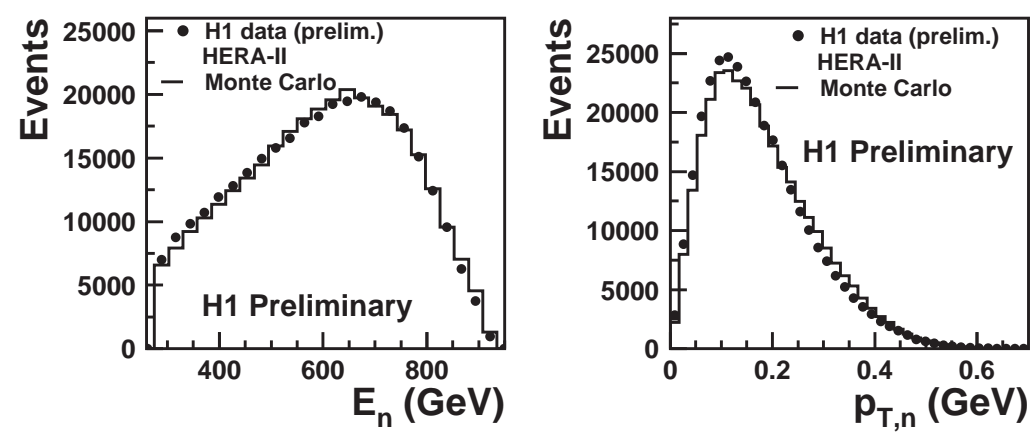

Figure 2: The observed neutron distributions from DIS interactions: (left) energy; (right) transverse momentum $p_{T}$. The data are compared to a weighted combination of RAPGAP $\pi^{+}$-exchange and DJANGO Monte Carlo simulations. 
The DJANGO program is used for the generation of standard DIS events. Leading neutron production via the pion exchange was simulated by the RAPGAP generator. The best description of the data is achieved if the predictions of the RAPGAP and DJANGO Monte Carlo event generators are combined, using some global weighting factors. The DJANGO and RAPGAP Monte Carlo simulations are calculated using GRV leading order parton distributions for the proton and the pion. The pion flux factor is taken from the light-cone representation of Holtmann et al. [5]:

$$
f_{\pi^{+} / p}\left(x_{L}, t\right)=\frac{1}{2 \pi} \frac{g_{p \pi n}^{2}}{4 \pi}\left(1-x_{L}\right) \frac{-t}{\left(m_{\pi}^{2}-t\right)^{2}} \exp \left(-R_{\pi n}^{2} \frac{m_{\pi}^{2}-t}{1-x_{L}}\right)
$$

where $g_{p \pi n}^{2} / 4 \pi=13.6$ and $R_{\pi n}=0.93 \mathrm{GeV}^{-1}$ is the radius of the pion-proton Fock state. In Fig. 2 comparisons of data with Monte Carlo for leading neutron spectra are shown.

The errors shown in the figures are calculated as the quadratic sum of the statistical and systematic errors. The average total error is about $11 \%$.

\section{Results}

Figure 3 shows the measurements of the semi-inclusive structure function $F_{2}^{L N}\left(Q^{2}, x, x_{L}\right)$ in the range $6 \mathrm{GeV}^{2}<Q^{2}<100 \mathrm{GeV}^{2}, 1.5 \cdot 10^{-4}<x<3 \cdot 10^{-2}, 0.32<x_{L}<0.95$ and $p_{T}<0.2 \mathrm{GeV}$ in comparison with the Monte Carlo simulations. For large values of $x_{L}$ ( $\gtrsim 0.7$ ), the RAPGAP $\pi^{+}$-exchange simulation describes the shape of the $x_{L}$ distribution

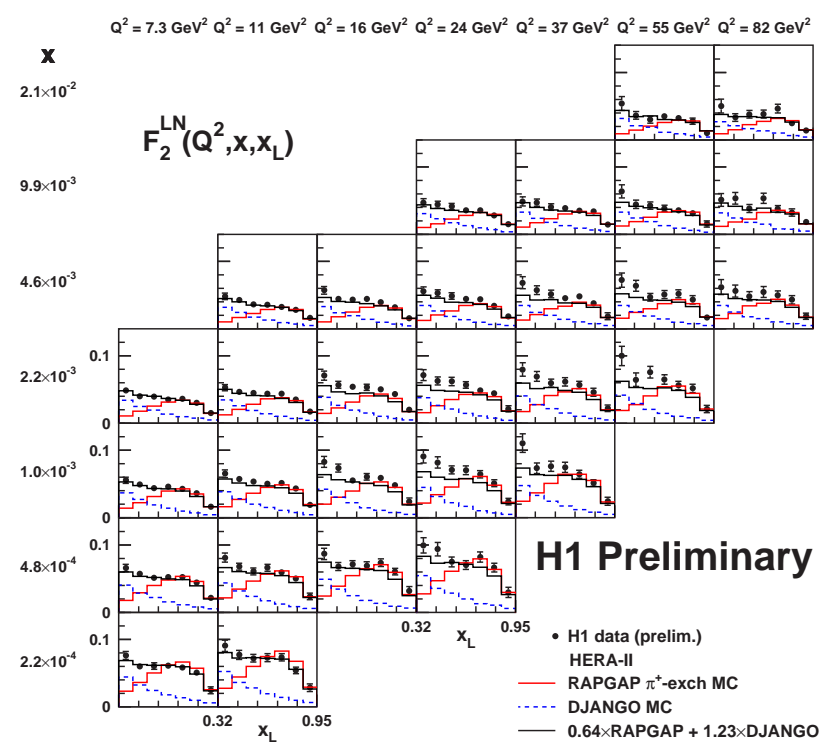

Figure 3: The semi-inclusive structure function $F_{2}^{L N(3)}$, for neutrons with $p_{T}<0.2 \mathrm{GeV}$, compared to the predictions of RAPGAP $\pi^{+}$-exchange (dashed line) and DJANGO (dotted line) Monte Carlo simulations. Also shown is a weighted combination of these two simulations (full line). 
well, in agreement with the assumption that at high $x_{L}$ the dominant mechanism for leading neutron production is pion exchange. The low-energy region and the overall distribution are well described by the weighted sum of RAPGAP $\pi^{+}$-exchange and the standard DIS Monte Carlo generator, DJANGO.

The factorisation properties of leading neutron events are tested by measuring the ratio of the semi-inclusive structure function $F_{2}^{L N}$ to the inclusive structure function $F_{2}$. This ratio is shown in Fig. 4 as a function of $Q^{2}$ in bins of $x$ and $x_{L}$. The values of $F_{2}$ are obtained from the H1-2000 parameterisation [6]. The ratios are almost flat and independent of $x$ and $Q^{2}$ in each $x_{L}$ bin implying that $F_{2}^{L N}$ and $F_{2}$ have a similar $\left(Q^{2}, x\right)$ behaviour.

Having established that pion exchange dominates leading neutron production at high $x_{L}$ and low $p_{T}$, the measurement of $F_{2}^{L N(3)}$ at $0.68<x_{L}<0.77$ and the integral over $t$ of the pion flux factor are used to estimate the pion structure function at low Bjorken- $x$. The measurement of $F_{2}^{L N(3)} / \Gamma_{\pi}$ can be interpreted as being equal to the structure function of the pion, where $\Gamma_{\pi}\left(x_{L}\right)=\int_{t_{0}}^{t_{\text {min }}} f_{\pi / p}\left(x_{L}, t\right) \mathrm{d} t$ is the integral of the pion flux over the mea-

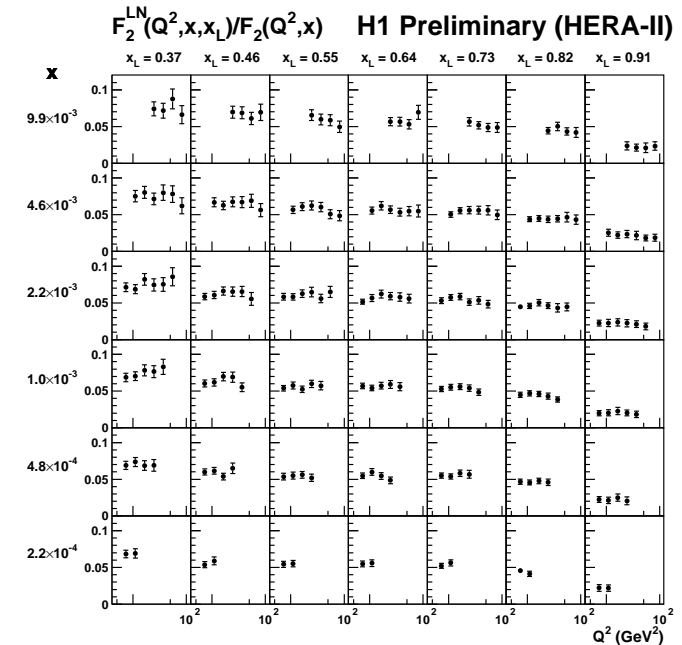

Figure 4: The ratio of the semi-inclusive structure function $F_{2}^{L N(3)}$, for neutrons with $p_{T}<0.2 \mathrm{GeV}$, to the inclusive structure function $F_{2}$ obtained from the $\mathrm{H} 1$ measurements of inclusive DIS [6]. sured $t$-range. The $t$-range is defined by the $p_{T}<0.2 \mathrm{GeV}$ range according to Eq. 1. The pion flux from Eq. 2 used for the RAPGAP simulation yields $\Gamma_{\pi}=0.131$ at $x_{L}=0.73$, which is the central value of the chosen $x_{L}$-bin.

The left-hand side of Fig. 5 shows $F_{2}^{L N(3)} / \Gamma_{\pi}$ as a function of $Q^{2}$ for fixed values of $\beta$ while the right-hand side of Fig. 5 shows $F_{2}^{L N(3)} / \Gamma_{\pi}$ as a function of $\beta$ for fixed values of $Q^{2}$. The data are compared to predictions of the parameterisations of the pion structure function GRV- $\pi$ [7] and ABFKW- $\pi$ [8]. The measurements are also compared to the H12000 parameterisation of the proton structure function [6] which is scaled by a factor $2 / 3$ in order to naively account for the different number of valence quarks in the pion and proton, respectively. The $Q^{2}$ distribution exhibits a rise with increasing $Q^{2}$ for all $\beta$ values in the measured range, which is similar in size and shape to that seen in the parameterisations of the inclusive structure functions of both the pion and proton. The $\beta$ distributions show a steep rise with decreasing $\beta$. This behaviour is in good agreement with the pion and proton structure function parameterisations. The comparison of $F_{2}^{L N(3)} / \Gamma_{\pi}$ and the pion structure function is affected by the uncertainty of the pion flux normalisation. Using other parameterisations of pion flux leads to values of the pion flux integral which may differ by up to $20 \%$. Moreover, the evaluation of the pion flux factor may depend on the absorptive corrections, which are not taken into account. The contributions from background processes, e.g. the exchange of $\rho$ and $a_{2}$-mesons and $\Delta$ production, are not taken into account. They are expected to affect only the absolute normalisation of the results. 

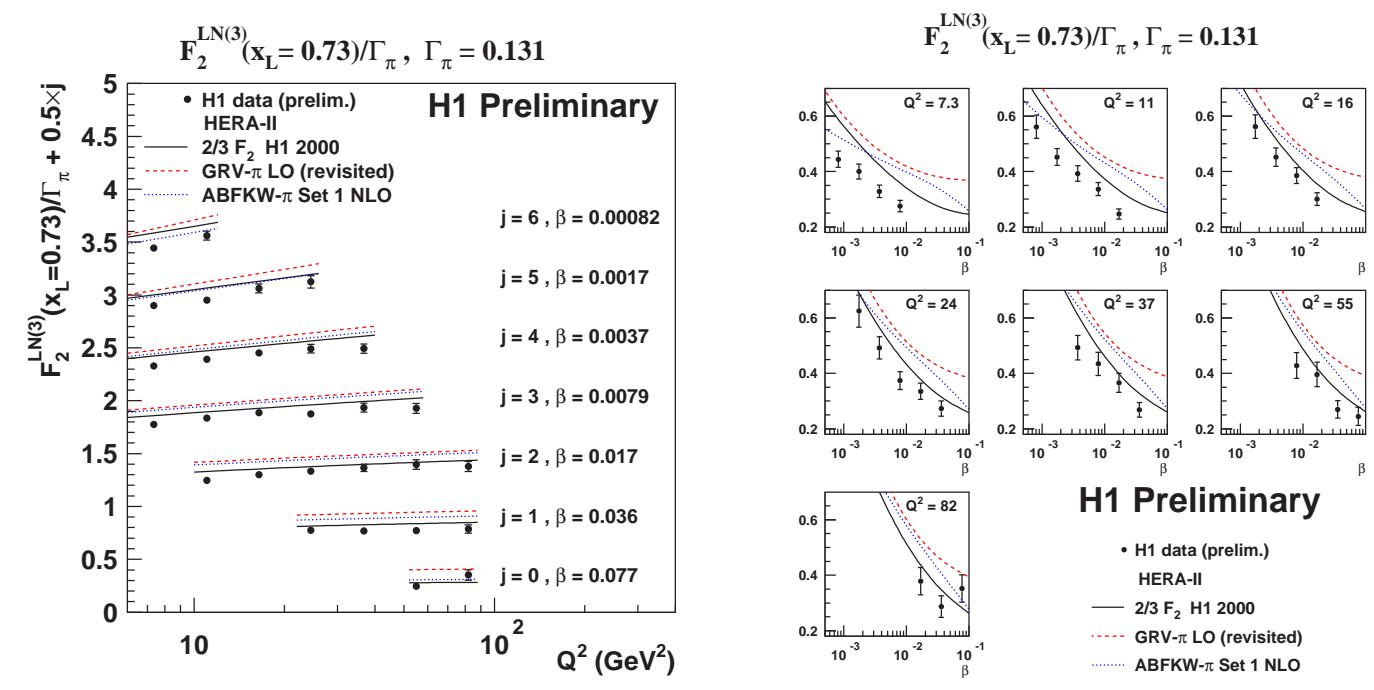

Figure 5: The semi-inclusive structure function $F_{2}^{L N(3)}$, for neutrons with $p_{T}<0.2 \mathrm{GeV}$, divided by the pion flux $\Gamma_{\pi}$ integrated over $t$ at the average value $x_{L}=0.73$. The pion flux is defined in Eq. 2.

\section{Summary}

The production of highly energetic forward neutrons has been studied in deep-inelastic positron-proton scattering. The semi-inclusive cross section $e p \rightarrow e n X$ has been measured in the kinematic region $6 \mathrm{GeV}^{2} \leq Q^{2} \leq 100 \mathrm{GeV}^{2}, 1.5 \cdot 10^{-4} \leq x \leq 3 \cdot 10^{-2}, 0.32 \leq x_{L} \leq 0.95$ and $p_{T}^{n} \leq 0.2 \mathrm{GeV}$. The measurements are well described by a mixture of the RAPGAP Monte Carlo program with the $\pi^{+}$exchange option and the DJANGO Monte Carlo program, which simulates inclusive DIS processes and uses the string fragmentation model. At $x_{L} \gtrsim$ 0.7 an acceptable description of the data is given by the RAPGAP $\pi^{+}$-exchange generator alone. The semi-inclusive structure function $F_{2}^{L N}$ and the inclusive structure function $F_{2}$ have similar $\left(x, Q^{2}\right)$ behaviour. The data are used to estimate the structure function of the pion.

\section{References}

[1] Slides: http: //indico. cern. ch/contributionDisplay $\cdot$ py? contribId=146\&sessionId=18\& conf Id=53294

[2] H1 Collaboration, A. Aktas et al., Eur. Phys. J. C 41, 273 (2005), [hep-ex/0501074].

[3] ZEUS Collaboration, S. Chekanov et al., Nucl. Phys. B 776, 1 (2007), [hep-ex/0702028].

[4] H1 Collaboration, H1prelim-08-111, http://www-h1.desy.de/h1/www/publications/htmlsplit/H1prelim-08-111.long.html

[5] H. Holtmann et al., Phys. Lett. B 338, 363 (1994).

[6] H1 Collaboration, C. Adloff et al., Eur. Phys. J. C 21, 33 (2001), [hep-ex/0012053].

[7] M. Glück, E. Reya and I. Schienbein, Eur. Phys. J. C 10, 313 (1999), [hep-ph/9903288].

[8] P. Aurenche, R. Baier, M. Fontannaz, M. N. Kienzle-Focacci and M. Werlen, Phys. Lett. B 233, 517 (1989). 